\title{
Operational momentum effect in children with and without developmental dyscalculia
}

\author{
Karin Kucian ${ }^{1,2 *}$, Fabienne Plangger ${ }^{1}$, Ruth O'Gorman $^{1,2,3}$ and Michael von Aster ${ }^{1,2,4,5}$ \\ ${ }^{1}$ Center for MR-Research, University Children's Hospital Zurich, Zurich, Switzerland \\ ${ }^{2}$ Children's Research Center, University Children's Hospital Zurich, Zurich, Switzerland \\ ${ }^{3}$ Center of Integrative Human Physiology, University of Zurich, Switzerland \\ ${ }^{4}$ Department of Child-and Adolescent Psychiatry, DRK-Hospital Westend Berlin, Germany \\ ${ }^{5}$ Clinical Psychology and Psychotherapy, University of Potsdam, Potsdam, Germany \\ *Correspondence: karin.kucian@kispi.uzh.ch \\ Edited by: \\ Klaus F. Willmes, RWTH Aachen University, Germany \\ Reviewed by: \\ Korbinian Moeller, Knowledge Media Research Center, Germany
}

Keywords: developmental dyscalculia, operational momentum, children, learning disability, numerical cognition, mental number line, symbolic calculation, attention

\section{A commentary on}

Examining the presence and determinants of operational momentum in childhood by Knops, A., Zitzmann, S., and McCrink, K. (2013). Front. Psychol. 4:325. doi: 10.3389/fpsyg.2013.00325

\section{THE PRESENCE OF OPERATIONAL MOMENTUM IN CHILDHOOD}

In their valuable article, Knops et al. (2013) challenge the existence of the operational momentum effect (OME) in children. The OME is characterized by the tendency to overestimate the result of addition problems and to underestimate the result of subtractions (McCrink et al., 2007). In line with previous findings, they replicated the OME in adults using a nonsymbolic approximation task. In contrast, children did not exhibit such an OME. This finding was quite unexpected since current studies claim that the OME is present in childhood, as early as 9 months of age (Pinhas and Fischer, 2008; McCrink and Wynn, 2009). Together with the evaluation of attentional orienting capacity, the authors concluded that an attentional shift along the mental number line (MNL) most probably explains the OME.

We take the liberty of adding own results in this commentary which further support these findings. We have also tested the OME in typically achieving children and a matched group with developmental dyscalculia (DD) (Table 1). Typically achieving children did not show an OME in a symbolic number line task, as children underestimated the location of results for both additions and subtractions on the number line (Figure 1). In contrast to the study demonstrating an OME in 9month old babies (McCrink and Wynn, 2009), we used a symbolic numerical task similar to the one used by Pinhas and Fischer (2008) who observed a reliable OME. However, their participants were already in adolescence. Therefore, it might be possible that since school-age children have lower experience in symbolic processing of calculations, an unconscious shift of attention on the MNL becomes evident only with increasing expertise and automatization.

Together with the findings of Knops et al., it seems that the left-right associations underlying the OME are dependent on development and experience. One might hypothesize that a complex interaction between visuo-spatial and attentional processes together with number related skills influence its development whereupon an early predisposition to relate representations of non-symbolic numerical magnitude to spatial length builds a core system (De Hevia and Spelke, 2010). In combination with cultural conventions such as reading direction, the experience of specific left-small/right-large associations (Berch et al., 1999; Opfer et al., 2010), numeric magnitude and number line estimation (Siegler and Booth, 2004) and the understanding of arithmetic concepts might lead first to the OME by non-symbolic presentation and later, after the acquisition of the symbolic number system, to the OME found by symbolic presentation (Pinhas and Fischer, 2008). That the OME is possibly subject to the dynamic nature of developing math proficiency would also be in line with current neuropsychological (Von Aster and Shalev, 2007) and neuronal models (Kucian and Kaufmann, 2009) suggesting the development of MNL representation successively depending on previous processes of representing numerical magnitudes by first verbal and later Arabic symbols. However, future studies will have to address these hypothesis systematically.

According to the assumption of such a hierarchical and interwoven model of OME, the impairment of one or several aspects may affect the development of the OME negatively.

\section{THE OME IN CHILDREN WITH DEVELOPMENTAL DYSCALCULIA}

DD is a specific learning disability of number processing and calculation. In addition to profound problems in numerical understanding, abnormalities in visuo-spatial, and attentional processes have also been associated with DD.

As expected, no OME was observed in our tested group of children with DD (Figure 1). Therefore, one might speculate that a lack of numerical understanding and reduced visuo-spatial and attentional functions, as often found in children with DD, might hinder the development of an OME.

\section{LIMITATIONS}

It is important to note that an adult control group is missing in our study. However, 
Table 1 | Demographic and testmetric data.

\begin{tabular}{|c|c|c|c|}
\hline & Dyscalculic children & Control children & $p$-value \\
\hline Handedness (right/ambidexter/left) & $14 / 1 / 1$ & $12 / 0 / 4$ & \\
\hline School grade & $3.1(1.1)$ & $2.9(0.9)$ & 0.604 \\
\hline General IQ & $99.0(6.9)$ & $110.1(7.0)$ & 0.000 \\
\hline Performance 10 & $100.0(12.2)$ & $108.6(10.2)$ & 0.038 \\
\hline Numeracy (percentile rank) & $14.7(20.1)$ & $68.9(18.8)$ & 0.000 \\
\hline Visuo-spatial memory span (block span) & $4.6(0.7)$ & $4.9(1.0)$ & 0.361 \\
\hline Visuo-spatial working memory (block span) & $2.1(0.7)$ & $2.8(0.9)$ & 0.043 \\
\hline
\end{tabular}

General IO was assessed by the mean of following subtests of the WISC-III (Wechsler, 1999): similarities, picture arrangement, calculation, block design, vocabulary. Performance 1Q, mean of picture arrangement, calculation, block design; Verbal IQ, mean of similarities, vocabulary. Numeracy was measured by the neuropsychological test battery for number processing and calculation in children ZAREKI-R (Von Aster et al., 2006). Visuo-spatial memory span was determined by the Corsi-Block-Tapping test (Corsi, 1972) and visuo-spatial working memory by the Corsi-Block-Suppression test (Beblo et al., 2004).

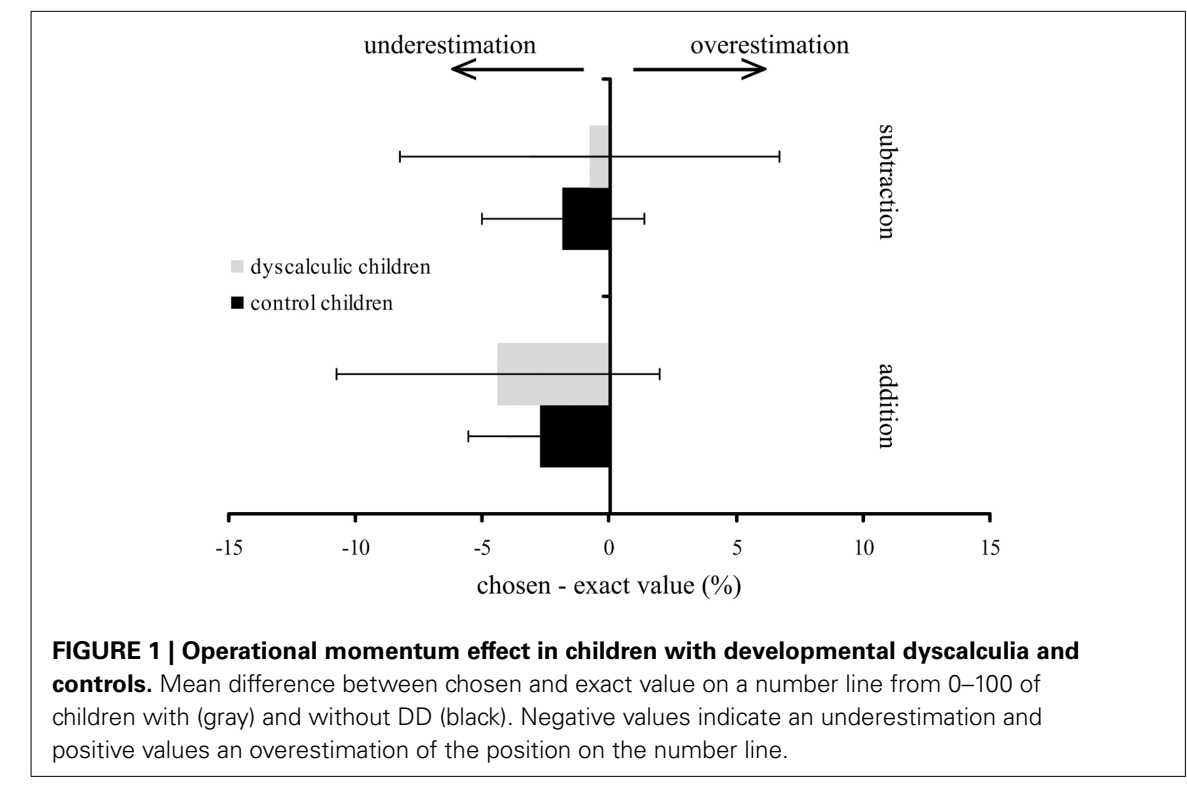

since Pinhas and Fischer (2008) demonstrated an OME in adolescents by means of a comparable paradigm to ours, we assume that the lack of OME in children is not due to differences in task design.

\section{DOES A FLAWED UNCOMPRESSION OF NUMERICAL INFORMATION CAUSE THE OME?}

Knops et al. argue that the lack of an OME during childhood speaks against a flawed compression-uncompression mechanism as the driving factor of the operational momentum bias, which implies that the OME is caused by a systematic bias during uncompression of a logarithmic representation of the MNL. Furthermore, number representations change from a logarithmic mapping to a linear mapping scheme during development and familiarity in a number range (Siegler and Opfer, 2003). Hence, the MNL representation cannot be assumed to be identical in children and adults examined by Knops. However, as outlined by the authors, if a flawed uncompression of numerical information causes the OME, it should be more pronounced in children who still represent numbers in a logarithmic fashion.

In contrast to the cohort of Knops et al., children in our study were older and showed a linear function describing the MNL representation like in adults, but exhibited no OME. Moreover, our results indicated that even after the completion of a specific number line training (Kucian et al., 2011), no OME was evident, although the training had a positive effect on the MNL representation. Therefore, our results further corroborate that differences in uncompression mechanisms of the MNL are unlikely to solely cause the OME.

\section{CONCLUSIONS}

Findings reported by Knops et al. and our study corroborate that the OME is not necessarily present in childhood and unlikely to be caused by flawed uncompression of the MNL. In addition, our results further point to a possible negative impact of DD on the development of the OME. In conclusion, the OME is probably dependent on development and a complex interaction of the maturity of numerical skills, visuospatial, and attentional processes as well as cultural conventions.

\section{ACKNOWLEDGMENTS}

Many thanks to all children and parents, who participated in this study and to the financial support by the NOMISFoundation and the German Federal Ministry of Education and Research (01GJ1011).

\section{REFERENCES}

Beblo, T., Macek, C., Brinkers, I., Hartje, W., and Klaver, P. (2004). A new approach in clinical neuropsychology to the assessment of spatial working memory: the block suppression test. J. Clin. Exp. Neuropsychol. 26, 105-114. doi: 10.1076/jcen.26.1.105.23938 
Berch, D. B., Foley, E. J., Hill, R. J., and Mcdonough Ryan, P. (1999). Extracting parity and magnitude from arabic numerals: developmental changes in number processing and mental representation. J. Exp. Child Psychol. 74, 286-308. doi: 10.1006/jecp.1999.2518

Corsi, P. M. (1972). Human memory and the temporal region of the brain. Diss. Abstr. Int. 34, 891.

De Hevia, M. D., and Spelke, E. S. (2010). Numberspace mapping in human infants. Psychol. Sci. 21, 653-660. doi: 10.1177/0956797610366091

Knops, A., Zitzmann, S., and Mccrink, K. (2013). Examining the presence and determinants of operational momentum in childhood. Front. Psychol. 4:325. doi: 10.3389/fpsyg.2013.00325

Kucian, K., Grond, U., Rotzer, S., Henzi, B., Schonmann, C., Plangger, F., et al. (2011). Mental number line training in children with developmental dyscalculia. Neuroimage 57, 782-795. doi: 10.1016/j.neuroimage.2011.01.070

Kucian, K., and Kaufmann, L. (2009). A developmental model of number representation. J. Behav. Brain Sci. 32, 340-341. doi: 10.1017/S0140525X09990069

McCrink, K., Dehaene, S., and Dehaene-Lambertz, G. (2007). Moving along the number line: operational momentum in nonsymbolic arithmetic. Percept. Psychophys. 69, 1324-1333. doi: 10.3758/BF03192949

McCrink, K., and Wynn, K. (2009). Operational momentum in large-number addition and subtraction by 9-month-olds. J. Exp. Child Psychol. 103, 400-408. doi: 10.1016/j.jecp.2009.01.013

Opfer, J. E., Thompson, C. A., and Furlong, E. E. (2010). Early development of spatial-numeric associations: evidence from spatial and quantitative performance of preschoolers. Dev. Sci. 13, 761-771. doi: 10.1111/j.1467-7687.2009.00934.x

Pinhas, M., and Fischer, M. H. (2008). Mental movements without magnitude? A study of spatial biases in symbolic arithmetic. Cognition 109, 408-415. doi: 10.1016/j.cognition.2008.09.003

Siegler, R. S., and Booth, J. L. (2004). Development of numerical estimation in young children. Child Dev. 75, 428-444. doi: 10.1111/j.14678624.2004.00684.x

Siegler, R. S., and Opfer, J. E. (2003). The development of numerical estimation: evidence for multiple representations of numerical quantity. Psychol. Sci. 14, 237-243. doi: 10.1111/1467-9280.02438

Von Aster, M., and Shalev, R. (2007). Number development and developmental dyscalculia. Dev. Med. Child Neurol. 49, 868-873. doi: 10.1111/j.14698749.2007.00868.x
Von Aster, M., Weinhold Zulauf, M., and Horn, R. (2006). ZAREKI-R (Neuropsychological Test Battery for Number Processing and Calculation in Children), revidierte Version. Frankfurt: Harcourt Test Services.

Wechsler, D. (1999). WISC-III Wechsler Intelligence Scale for Children. Bern, Göttingen, Toronto, Seattle: Hans Huber.

Received: 24 April 2013; accepted: 25 October 2013; published online: 12 November 2013.

Citation: Kucian K, Plangger F, O'Gorman $R$ and von Aster M (2013) Operational momentum effect in children with and without developmental dyscalculia. Front. Psychol. 4:847. doi: 10.3389/fpsyg.2013.00847

This article was submitted to Developmental Psychology, a section of the journal Frontiers in Psychology.

Copyright (C) 2013 Kucian, Plangger, O'Gorman and von Aster. This is an open-access article distributed under the terms of the Creative Commons Attribution License (CC BY). The use, distribution or reproduction in other forums is permitted, provided the original author(s) or licensor are credited and that the original publication in this journal is cited, in accordance with accepted academic practice. No use, distribution or reproduction is permitted which does not comply with these terms. 